\title{
Impact of Urinary Tract Infection on Steroid Response in Idiopathic Nephrotic Syndrome in Children
}

\author{
Tanuka Barua' \\ Razia Sultana ${ }^{2}$ \\ Pradip Kumar Datta ${ }^{3}$ \\ Jhulan Das Sharma ${ }^{4}$ \\ Md Rezaul Karim ${ }^{5^{*}}$ \\ Mainuddin Ahmad ${ }^{6}$
}

'Department of Pediatrics

Chattagram Maa-O-Shishu Hospital Medical College

Chittagong, Bangladesh.

${ }^{2}$ Shishu Bikash Kendra

Chittagong Medical College

Chittagong, Bangladesh.

${ }^{3}$ Department of Nephrology Chittagong Medical College

Chittagong, Bangladesh.

${ }^{4}$ Department of Pediatrics Southern Medical College

Chittagong, Bangladesh.

${ }^{5}$ Department of Pediatrics

Chittagong Medical College

Chittagong, Bangladesh

${ }^{6}$ Department of Pediatrics Dhaka Medical College

Dhaka, Bangladesh.

\section{*Correspondence to:}

\section{Dr. Md Rezaul Karim}

Professor of Pediatrics

Chittagong Medical College

Chittagong, Bangladesh.

Mobile : +8801733556177

E-mail :drkarim78@yahoo.com

\begin{abstract}
Context: UTI is one of the most common infection in nephrotic syndrome and may be a cause of delayed steroid response. Objective:To observe the impact of urinary tract infection on steroid response in idiopathic nephrotic syndrome children aged 2-6 years. Study design: Quasi experimental study Study period \& place: Pediatric ward of Chittagong Medical College Hospital, Chittagong from 01.01.2009 to 31.12.2009. Participants: 52 Nephrotic syndrome children aged 2-6 years with typical clinical features Group A: Nephrotic syndrome with UTI, Group B: Nephrotic syndrome without UTI. Methods: Heat coagulation test, urine for R/M/E and $\mathrm{C} / \mathrm{S}$ was done in every patient. Steroid was given according to standard regimen. Date of starting of steroid was recorded. Antibiotic was given in group A cases according to $\mathrm{C} / \mathrm{S}$ report. Patients were followed for clinical and urinary remission. Group A and B were compared for remission time achieved by statistical method. Results: A male preponderance was noted about 57.7\% against female about $42.3 \%$. Generalized swelling of body \& scanty micturation found in cent percent study group. Ascitis was found in 23.08\%. Scrotal/labial swelling 7.69\%.UTI developed in $30.8 \%$ of patients of NS. Male female ratio is $1: 1$. Infection delayed the remission of proteinuria. Mean remission time of NS without UTI patients was 7.39 days and with UTI patients was 9.31 days. In statistical analysis, mean remission time in group $\mathrm{A}=9.31+2.24$ days $($ mean $+\mathrm{SD})$, in group $\mathrm{B}=7.39+2.51$ days $($ mean $+\mathrm{SD}), \mathrm{P}$ value $=<0.05$, statistically significant. Conclusion: UTI in nephrotic syndrome causes delayed remission of proteinuria and may be asymptomatic.It should be screened in every nephrotic syndrome children routinely.
\end{abstract}

Key words : Nephrotic syndrome; UTI; proteinuria.

\section{INTRODUCTION}

Nephrotic syndrome is a common chronic disease in childhood. Incidence of idiopathic nephrotic syndrome in western countries varies between 2-7new cases with prevalence rates of nearly 16 cases per $100000^{1-2}$. A study at Bangabandhu Sheikh Mujib Medical University (BSMMU) showed that 50-60\% of total indoor bed in pediatric renal unit is occupied by the patient of nephrotic syndrome ${ }^{3}$.

Children of nephrotic syndrome are exposed to a variety of infectious complication that result in significant mortality and morbidity especially in developing countries such as ours $^{4-5}$. Of all infection UTI are of special interest because of their association with propensity for long term damage and in most cases they are asymptomatic $^{6-7}$. Information regarding there prevalence in nephrotic syndrome and the extent of impact on steroid response is scant and conflicting. This study add information about the impact of UTI on steroid response in nephrotic syndrome for early diagnosis of UTI to prevent related hazards. 


\section{MATERIALS \& METHODS}

Heat coagulation test, urine $\mathrm{R} / \mathrm{M} / \mathrm{E}$ and $\mathrm{C} / \mathrm{S}$ was done in every patient. Steroid was given according to standard regimen ${ }^{8}$. Date of starting of steroid was recorded. Antibiotic was given in culture positive cases according to $\mathrm{C} / \mathrm{S}$ report. Patients were followed for clinical and urinary remission. Culture positive and negative cases were compared for remission time achieved by statistical method.

\section{RESULTS \& DISCUSSION}

It was a quasiexperimental study.In this study ,male preponderance was noted in $52.2 \%$ cases like other studies ${ }^{3}$. But reasons for male preponderance was obscure.Cent percent patients presented with generalized swelling and scanty micturition followed by $23.8 \%$ with ascities and $7.69 \%$ with scrotal swelling.

Prevalence of UTI in our study was $30.8 \%$ that correlates with previous studies. But both male and female were equally infected with UTI which is not supported by many other studies 9 . Majority of UTI patients (68.8\%) was asymptomatic which is consisted with standard reference ${ }^{10-11}$. Regarding clinical presentation of UTI, we had found fever(60\%), lower abdominal pain (20\%), dysuria(20\%). As we know typical presentation of UTI are dysuria,loin pain \& generalized symptoms like fever,anorexia,abdominal pain,vomiting ${ }^{12}$. Our study is consisted with these findings.

In our study,pyuria was found in $62.5 \%$ culture positive cases and in $52.8 \%$ culture negative cases showing poor correlation between pyuria and definite UTI.This is consisted with observation of M Rahman,K M Rahman'.

Causative organism of UTI isolated in our study were E coli in $50 \%$,klebsiella in $25 \%$,coliforms \& proteus in $18.7 \%$ \& $6.3 \%$ respectively. According to Gulati, Gupte et al,E coli is the commonest organism followed by klebsiella ${ }^{13}$. Our findings is supported by that study.

Table 1: Common organisms of UTI

\begin{tabular}{lcc} 
Organisms & Frequency & Percentage $(\%)$ \\
E. Coli & 08 & 50.0 \\
Klebsiella & 04 & 25.0 \\
Coliform & 03 & 18.7 \\
Proteus & 01 & 6.3 \\
Total & 52 & 100.0 \\
\hline
\end{tabular}

The mean remission time of proteinuria was 7.39 days in patients of nephrotic syndrome without UTI \& 9.31 days in nephritic syndrome with UTI patients showing delayed remission UTI patients which is consisted with other studies ${ }^{14,15}$.

Table 2: Remission time in nephritic syndrome with UTI and without UTI patients. (with $\mathrm{t}$ - test significance)

\begin{tabular}{|c|c|c|c|c|c|c|c|}
\hline & & $\mathrm{N}$ & Mean & $\pm \mathrm{SD}$ & Median & Range & Sign. \\
\hline \multirow{4}{*}{$\begin{array}{l}\text { Remission } \\
\text { Time } \\
\text { (Days) }\end{array}$} & Group A (UTI) & 16 & 9.31 & 2.24 & 9.50 & $6-13$ & $\mathrm{P}<0.05$ \\
\hline & & & & & & & Significant \\
\hline & Group B ( $\mathrm{N}_{0}$ UTI) & 36 & 7.39 & 2.51 & 7.00 & $4-18$ & \\
\hline & TOTAL & 52 & 7.98 & 2.57 & 8.00 & $4-18$ & \\
\hline
\end{tabular}

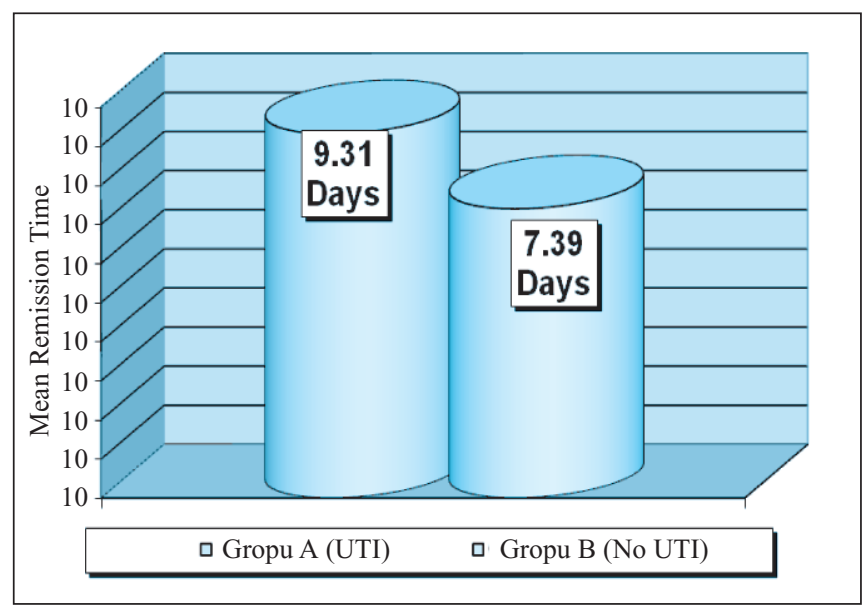

\section{CONCLUSION}

Urinary tract infection in nephritic syndrome is mostly asymptomatic and causes delayed remission of proteinuria.Every nephrotic child should be screened for UTI routinely for proper management and to avoid prolonged hospital stay, thereby to prevent long term renal damage.

\section{DISCLOSURE}

All the authors declared no competing interest. 


\section{REFERENCES}

1. H. M. Nanjundaswany, K.D. Phad ke. Steroid sensitive Nephrotic syndrome. Indian J of Pediatr. 2002; 69: 1059-1063.

2. Consensus statement on management and audit potential for steroid responsive nephrotic syndrome -Report of a workshop by the British Association for Paediatric Nephrolozy and Research Unit, Royal College of Physicians. Arch Dis Child. 1994;70: 151-1573.

3. Hossain M M, Ara H, Khan M R. A study of nephrotic syndrome in children at IPGMR. Bang Peditr. 1982; 6(1): 25- 28.

4. P senguttuvan, K Ravanan, N Pravu, V Tamilarasi. Infection encountered in childhood nephrotics in a paediatric renal unit. Indian J Nephrol.2004 ; $14: 85-88$

5. R N Srivastava, A Moudgil, O Khurana. Serious infections and mortality in nephrotic syndrome. Indian pediatr. 1987 ; $24: 1077-1080$.

6. M Uhari, M Nuutinen. Epidemiology of symptomatic infection of the urinary tract in children. Br Med J. 1988 ; 297 : 450-452.

7. R K Alwadhi, J L Mathew, B Rath. Clinical profile of children with nephrotic syndrome not on glucocorticoid therapy but presenting with infection. J Paediatr Child Health. 2004 ; $40: 28-32$.

8. CR Kothary. Research Methodology- Methods and Technique. 2nd ed. New Delhi: New Age Int P Ltd; 2004; 176-180.

9. M Rahman, K M Rahman. Urinary tract infection in school children. BMRC Bulletin. 1979; 2: 67-70.

10. Murani K N, Khan K M, Ramzan A. Infection in children with nephritic syndrome.J Coll Physicians Surg Pak.2003;13(6):337-339.

11. S Gulati, V Kher, A Gupta, P Arora, P K Rai, R K Sharma. Spectrum $\quad$ of infection of Indian children with nephrotic syndrome. Pediatr Nephrol. 1995; 06: 431-434.

12. George H, McCracken J R, Diagnosis and management of acute urinary tract infection in infants and children. The pediatr Infect Dis J. $1987 ; 6: 107-112$.

13. S I Adeleke, M O Asani. Urinary tract infection in children with nephritic syndrome in Kano, Nigeria. Annals of African Medicine.2009;8(1):38-41.

14. S Gulati V kher, A Arora, S Gupta, S Kale. Urinary tract infection in nephrotic syndrome. Pediatr Infect Dis J. 1996 ; 15 : 237-240.

15. Emilia M, H Koch, M D Fujimura, Y Okay. Influence of nephroitic state on the infectious profile in childhood idiopathie nephrotic syndrome. Rev Hosp clinc Fac Med. S Paulo. 2004; 59 (5):273-274. 